\title{
Smart Parking Using Lifi
}

\author{
Nikhil Belhekar' ${ }^{1}$, Vishakha Dhamdhere ${ }^{2}$, Anurag Autade ${ }^{3}$, Abhishek Darekar $^{4}$, \\ Prof. Ravikiran Suryawanshi ${ }^{5}$ \\ Student, Comp Dept, Trinity College of Engineering and Research, Pune, India ${ }^{1,2,3,4}$ \\ Guide, Comp Dept, Trinity College of Engineering and Research, Pune, India ${ }^{5}$
}

\begin{abstract}
The LI-FI is the newest technology in the Field of wireless communication. Nowadays many people are using internet to fulfil their task through wired or wireless technologies. As the number of users is increasing, the rate of data transmission in the wireless network automatically decreases. WI-FI provides us speeds near about $150 \mathrm{mbps}$ as per IEEE 802.11n but still it is not able to fulfil the requirement of the user because of such reasons we are introducing LIFI. According to the German psychist Harald Hass LI-FI provides more speed (10megabits per second minimum) data transmission by using visible light. So here in this condition we are analysing the LI-FI/WI-FI. It's the same idea band behind infrared remote controls but far more powerful. Haas says his invention, which he calls D-LIGHT, can produce data rates faster than our average broadband connection. Recently, parking vehicle is most tedious job. Hence, in order to solve this problem, a reliable system is proposed. our system solves the current parking problems by offering guaranteed parking reservations with the lowest possible cost and searching time for drivers and the highest revenue and resource utilization for parking managers.
\end{abstract}

Keywords: Dynamic Pricing, Dynamic Resource Allocation, Li-Fi, Smart Car Parking

\section{INTRODUCTION}

LiFi is a wireless optical networking technology that uses Light-Emitting Diodes (LEDs) for data transmission. LiFi is designed to use LED light bulbs similar to those currently in use in many energy-conscious homes and offices. highspeed wireless communication technology that uses visible light (presently using LEDs) to transmit information. Using LiFi they check if the parking slot are empty or not. If parking slot are empty then it will park the car.

\section{LITERATURE SURVEY}

\section{A comparative Study of Wireless Protocols with Li-Fi Technology}

The Li-Fi is visible light communication (VLC) technology, developed by scientists Hass at University of Edinburg and deals with data transmission through LED light bulb that is over IEEE 802.157.7 standards used in personal area network (PAN). They explained as, "if LED is ON, transmit a digital data 1 and likewise if the LED is OFF, transmit a digital 0 or null or simply no data transmission happens". The Wi-Fi gives us to speed up to $150 \mathrm{mbps}$ as per IEEE 802.11.n and directly connected computer to computer with the suitable network. Bluetooth devices of the application is a cordless mouse, keyboard, and hands-free headset. UWB is the high-bandwidth multi-communication. ZigBee is reliable for wirelessly networked monitoring and control networks.

\section{LI-FI Technology for Data Transmission through LED}

According to the German psychist Harald Hass LI-FI provides more speed (10megabits per second) data transmission by using visible light. So here in this condition we analyse the LI-FI/WI-FI. It's the same idea band behind infrared remote controls but far more powerful. Haas says his invention, which he calls D-LIGHT, can produce data rates faster than our average broadband connection. In this we are going to compare and analyse the speed of LI-FI and WI-FI and also network jamming problem during the increase the increasing of users.

\section{Li-Fi A New Generation Wireless Communication}

Li-Fi comprises a wide range of frequencies and wavelengths, from the infrared through visible and down to the ultraviolet spectrum. It includes sub-gigabit and gigabit-class communication speeds for short, medium and long ranges, and unidirectional and bidirectional data transfer using line-of-sight or diffuse links, reflections and much more. It is not limited to LED or laser technologies or to a particular receiving technique. Li-Fi is a framework for all of these providing new capabilities to current and future services, applications and end users. 


\section{International Journal of Advanced Research in Computer and Communication Engineering}

Vol. 7, Issue 11, November 2018

\section{PROPOSED SYSTEM}

In Proposed system we are analysing the parking issue which may happens due following Reasons:

1) Most parking lots are not even marked properly.

2)Most of the parking is managed but not organised.

3) Problem of parking on special occasions.

From all these parameters we are plotting graph to analyse data to show the high parking issues.

\section{SYSTEM ARCHITECTURE}

Following diagram is our system's architecture diagram:

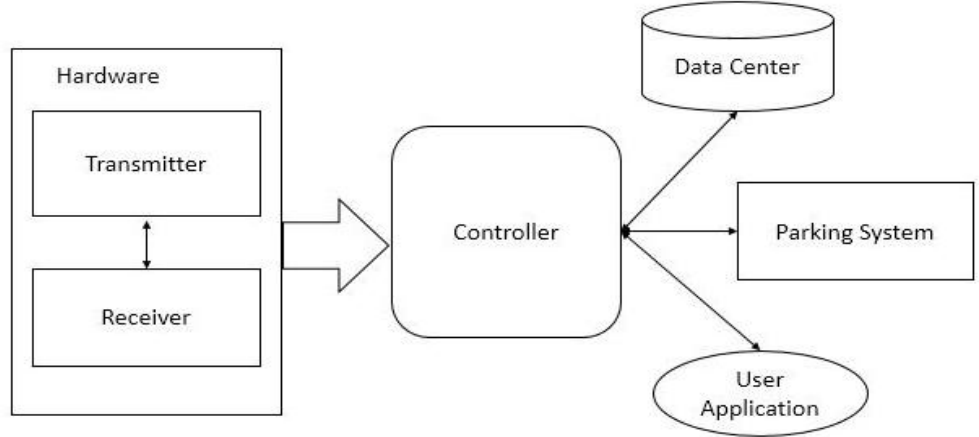

figure 1: system architecture

In this system we are taking input from hardware like sensors, controllers. The data collected by hardware gets uploaded through WIFI on cloud. After analysing uploaded data user can see the graph according to area or roads.

\section{METHODOLOGIES}

We are going to use transmitter, receiver, controller, a parking system and a database. Therefore, the study consisted of controllers, user application and decision-making algorithms for analysis of large collected data from sensors. A transmitter is a different kind of antennas that does the opposite job to a receiver: it turns electrical signals into radio waves so they can travel sometimes thousands of kilometres around the Earth or even into space and back. The purpose of most transmitter is radio communication of information over a distance. Combines the information signal to be carried with the radio frequency signal which generates the radio waves. The information of transmitter and receiver send the information of the controller. Then using User Application, it checks the parking slot for an auto car. The user data can be stored in database. A controller, in a computing context, is a hardware device or a software program that manages or directs the flow of data between two entities. So, the hardware data send the user using controller.

\section{VI.ADVANTAGES}

- We can easily identify if Parking slots are empty or not.

- It will help to reduce the time in the parking.

\section{CONCLUSION}

Cars can check Parking slots. It saves the data to the cloud and check the parking slot. If slot is empty, car can be parked at the empty slot.

\section{REFERRENCES}

[1]. Manas Ranjan Mallick," A Comparative Study Of Wireless Protocols With Li-Fi Technology: A Survey”, International Journal of Advanced Computational Engineering and Networking, Volume-4, Issue-6, Jun.-2016.

[2]. Mr. Shailendra Yadav, Mr. Pradeep Mishra, Miss. Minakshee Velapure, Prof.P.S.Togrikar, "LI-FI Technology for Data Transmission through LED”, Imperial Journal of Interdisciplinary Research (IJIR),Vol-2, Issue-6, 2016.

[3]. Varsha Shrivastava, Neha Malik, "Li-Fi-A New Generation Wireless Communication", IJLTEMAS, Volume III, Issue V, May 2014.

[4]. Karabi Deka, Parthana Bora, Pritam Kr. Goswami, Bhargobi Borah, Deepjyoti Chakraborty, "Design of a Li-Fi based Data Transmission System", International Journal of Innovative Research in Science, Engineering and Technology, Vol. 6, Issue 9, September 2017.

[5]. Shirke Suraj .V, Dalvi Sagar .D, Ligade Pankaj .S, "Li-Fi: Data Transmission through Light",International Journal of Innovative Research in Computer and Communication Engineering,Vol. 9,Issue 3,March 2016. 